\title{
STABILITY ANALYSIS OF SEMI-ACTIVE INERTER-SPRING-DAMPER SUSPENSIONS BASED ON TIME-DELAY
}

\author{
Changning LiU \\ School of Automotive and Traffic Engineering, Jiangsu University, Zhenjiang, China \\ Long Chen, Xiaoliang Zhang \\ Automotive Engineering Research Institute, Jiangsu University, Zhenjiang, China \\ e-mail: chenlong@ujs.edu.cn \\ XIAOFENG YANG \\ School of Automotive and Traffic Engineering, Jiangsu University, Zhenjiang, China
}

\begin{abstract}
To analyze the variation rule of the critical instability time-delay of the semi-active ISD suspension, a general solution was proposed and applied into two three-element-structure ISD suspensions. The dynamical response and the performance of the semi-active ISD suspension in various time-delays indicated that different structures had different suspension performance. The time-delay deteriorated the performance of all of the suspensions which were analyzed. The stability of the semi-active ISD suspension would seriously weaken with the critical time-delay. This paper provided a general solution for parameters selection in semi-active ISD suspension design to avoid the negative effect of the time-delay.
\end{abstract}

Keywords: semi-active suspension, time-delay, controllable inerter, stability, ride comfort

\section{Introduction}

The semi-active suspension, consisting of adjustable stiffness spring or adjustable damping, is a form of controllable suspension (Yin et al., 2016; Sapiński, 2005; Sun et al., 2016; Zuo and Slotine, 2005; Kęcik and Warmiński, 2011). This suspension can adjust the spring or damping parameters according to different driving conditions. So, a best trade off between comfort and safety can be met at a given time. Semi-active control adapts to different driving conditions, which can better take into account different requirements of ride comfort and safety. However, the damper or stiffness adjustable semi-active suspension, like the traditional passive suspension, is a spring-damper (SD) structure which is based on the classical vibration isolation theory. This structure limits further improvement of the suspension performance.

The proposal of an inerter and a structure of the inerter-spring-damper (ISD) suspension break through the limit of the traditional spring-damper structure (Smith, 2002; Jiang and Smith, 2015; Chen and Smith, 2015; Gonzalez-Buelga et al., 2015; Shen et al., 2017; Hu et al., 2018). The inerter is a two-terminal device in which the forces applied at the terminals are equal, opposite and proportional to relative acceleration between the nodes. As for the structure of inerter-spring-damper is more complex than the traditional spring-damper structure, there are different kinetic features for manifold ISD suspension structures (Chen et al., 2017). With the addition of control (Hu et al., 2016), the time-delay of the semi-active ISD suspension will be non-negligible. Stability is the most important issue with control systems and the primary requirement of a qualified system. The time-delay is mainly caused by the measurement delay of the sensor, the calculation delay of the control law and the delay of the actuator. The negligence of the time-delay may worsen the control ability. Luckily, the inertial force control has more advantages than the damping force control. The damping force control requires a speed signal 
for controlling. However, the inertial force control is only related to acceleration. In most practical systems, the speed signal cannot be directly obtained, but acquisition of the acceleration signal is direct and accurate (Estrada et al., 2014). Therefore, inertial force control is simpler and accurate than damping force control.

In recent years, the analysis and design of time-delay systems becomes a research boiling spot in the research community. A large number of new academic fruits are obtained in the application as well as theory fields. For example, Han et al. (2017) analyzed a non-linear active suspension with an executive body in time-delay. It is a SD structure which is fixed, so the time-delay only has one possibility. Eller et al. (1969) proposed an optimal strategy to reign a linear time-delay system. Sipahi and Olgac (2006a) demonstrated that stability maps of a linear time invariant retarded time delayed systems (LTI-TDS). Vyhlidal and Zitek (2009) used the Mikhaylov criterion to neutral time delay systems. Strecker et al. (2015) studied three different semi-active strategies and compared them. The response time of the MR damper and the by-pass valve have a serious impact on the ability of the semi-active suspension. While, that work is just dedicated to the conditional semi-active suspension whose structure is fixed and the ability is as close to the theoretical limit as possible. The topology structure of the inerter-spring-damper is more complex, and it is an effective approach to enhance the ability of the suspension.

This paper provides a general solution for stability analysis of the semi-active ISD suspension. This solution can explore the variation rule of the critical instability time-delay of the semi-active ISD suspension with the inerter coefficient and the unsprung mass. In Section 2, the general solution for solving the critical instability condition and the variation rule of the critical instability time-delay of the semi-active ISD suspension with vehicle parameters are proposed. In Section 3, two suspension structures with the skyhook control strategy, easy to realize in engineering, are analyzed. The quarter-car model of semi-active ISD suspension with time-delay is established and the time-delay differential equation of the semi-active ISD suspension is deduced. Then, by a numerical simulation, the variation law of the critical instability timedelay of the semi-active ISD suspension with the inerter coefficient is analyzed. In Section 4, the optimal parameters of the suspension selected by the previous numerical analysis are used to build the suspension model. The kinetic response and the performance of the semi-active ISD suspension in different time-delays, compared with the traditional passive suspension, are investigated under the condition of random incentive.

\section{A general solution for stability analysis of the semi-active ISD suspension}

In order to ensure that the semi-active ISD suspension can work under effective conditions, the sum of the time-delay for all the sensors, processor and actuator should be limited to the critical time-delay. If the time-delay is out of the critical time-delay, the control of the semi-active ISD suspension is invalid. So the critical time-delay of the semi-active ISD suspension should be solved before designing the suspension control system. For the semi-active ISD suspension, the topology of the element inerter, spring and damper is very diverse and complex. A general solution for stability analysis to solve the critical time-delay and analyze the variation rule of the critical instability time-delay with the inerter coefficient and the unsprung mass is necessary. Here are the steps of this solution:

Step 1: Establishing the kinetic semi-active ISD suspension model with time-delay and getting kinetic equations. The structure of the semi-active ISD suspension is not always first-order. More generally, the structure is high-order. For a high-order structure, the kinetic equations are also pluralistic.

Step 2: Solving the kinetic equations. Those kinetic equations are linear differential equations with time-dependent constant coefficients. The characteristic equations and eigenroots can 
be solved. For the first-order structure, those equations can be easily solved. But equations for a high-order structure may be transcendental equations. In this condition, the Taylor series expansion will help to solve the problem.

Step 3: Judging the stability condition and solving the critical time-delay. According to the Lyapunov stability criterion of invariant linear differential equations with constant coefficients, the sufficient and necessary condition for the asymptotic stability of the system with time-delay is that all the eigenroots of the system lie in the left portion of the complex plane. In addition, the variation rule of the critical instability time-delay of the semi-active ISD suspension with the vehicle parameters can be obtained.

Step 4: If the critical time-delay is long enough, the vehicle parameters can be designed based on the above solution results. If the critical time-delay does not exist, the suspension system is unstable. The suspension structure should be redesigned.

Step 5: Simulation verification. Testing of the semi-active ISD suspension to ensure that the solving results are accurate. It is also an important progress for the semi-active ISD suspension designed.

\section{Application of the general solution's in three-element semi-active ISD suspension}

\subsection{Quarter-car kinetic model of semi-active ISD suspension}

The most basic ISD suspension structure is the three-element-structure and it is most frequency easily used in the engineering practice (Chen M.Z.Q. et al., 2012; Chen L. et al., 2014). Considering that the elastic element is indispensable for the vehicle suspension system to maintain the vehicle mass, the three-element-structure ISD suspension only has two topological structures. One is a spring, a damper and an inerter connecting the body and the wheel respectively (S1). The other one is a spring connecting the body and the wheel, but the damper and the inerter are connected in series and then in parallel with the spring (S2). The two kinds of the three-element quarter-car kinetic model of the semi-active ISD suspension are illustrated in Fig. 1.

(a)

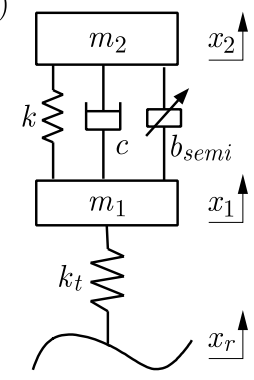

(b)

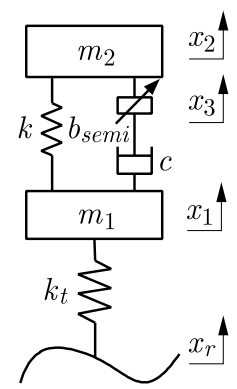

Fig. 1. Quarter-car kinetic model of semi-active ISD suspension; (a) S1, (b) S2

The skyhook control strategy is a classic control method and the control model is simple and easy to build, simple to calculate, with less application state and convenient measurement. The skyhook control strategy is utilized into the controllable inerter to improve the performance of the suspension. The effect of controllable inerter can be equivalent to an inertial force $F_{\text {semi }}$. And the force for S1 and S2 structure can be denoted as

$$
F_{\text {semi }}=\left\{\begin{array}{lll}
b_{\text {emi }}\left(\ddot{x}_{2}-\ddot{x}_{1}\right) & \text { for } & \mathrm{S} 1 \\
b_{\text {semi }}\left(\ddot{x}_{2}-\ddot{x}_{3}\right) & \text { for } & \mathrm{S} 2
\end{array}\right.
$$


Because the model of the semi-active ISD suspension is a passenger car, the main evaluation index is riding comfort. The control strategy of semi-active ISD suspension is comfortable-oriented. The control model for the semi-active ISD suspension system is illustrated in Fig. 2. The control model can be divided into two parts: the semi-active ISD suspension controller and semi-active ISD suspension system. The model was stimulated by pavement excitation, and the states were sampled by sensors and acted as an input to the semi-active ISD suspension controller. The controller first generated an ideal control force $F_{\text {semi }}$ under the law of the control strategy. Then, the classification model was applied to generate a realizable control force with velocity of rattle space. And the parameter $b_{\text {semi }}$ of structure S1 was following the criteria as

$$
b_{\text {semi }}= \begin{cases}b_{\max } & \ddot{x}_{2}\left(\ddot{x}_{2}-\ddot{x}_{1}\right) \geqslant 0 \\ b_{\min } & \ddot{x}_{2}\left(\ddot{x}_{2}-\ddot{x}_{1}\right)<0\end{cases}
$$

Equation (3.2) is to make the controllable inerter transfer the maximum force when the controllable inerter has the same function as the ideal skyhook inerter, and transfer the minimum force when the forces are in opposite direction. This means that the smallest inerter coefficient $b_{\text {min }}$ is always desirable, and the ideal state is $b_{\min }=0$.

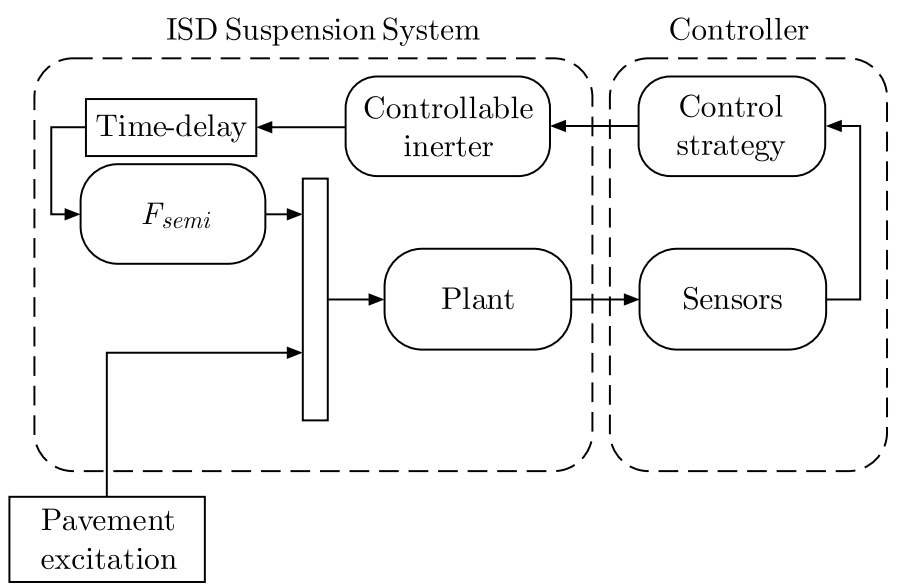

Fig. 2. Schematic block diagram of semi-active ISD suspension control strategy

And the following equations are kinetic equations for the three elements semi-active ISD suspension of the quarter model for a vehicle with time-delay. The parameter $b_{\text {semi }}$ is the semi-active controllable inerter coefficient. In practice, the inertia always exists, so $b_{\text {semi }}$ cannot be 0 . It can be replaced with $b_{0}$ and $b_{r}$. $b_{0}$ is the base value of the inerter coefficient; $b_{r}$ is the controllable inerter coefficient. Equations (3.3) is for structure S1 and Eqs. (3.4) is for structure S2

$$
\begin{aligned}
& m_{1} \ddot{x}_{1}+k_{t}\left(x_{1}-x_{r}\right)-k\left(x_{2}-x_{1}\right)-b_{0}\left(\ddot{x}_{2}-\ddot{x}_{1}\right)-b_{r}\left[\ddot{x}_{2}(t-\tau)-\ddot{x}_{1}(t-\tau)\right] \\
& \quad-c\left(\dot{x}_{2}-\dot{x}_{1}\right)=0 \\
& m_{2} \ddot{x}_{2}+k\left(x_{2}-x_{1}\right)+b_{0}\left(\ddot{x}_{2}-\ddot{x}_{1}\right)+b_{r}\left[\ddot{x}_{2}(t-\tau)-\ddot{x}_{1}(t-\tau)\right]+c\left(\dot{x}_{2}-\dot{x}_{1}\right)=0
\end{aligned}
$$

and

$$
\begin{aligned}
& m_{1} \ddot{x}_{1}+k_{t}\left(x_{1}-x_{r}\right)-k\left(x_{2}-x_{1}\right)-c\left(\dot{x}_{3}-\dot{x}_{1}\right)=0 \\
& m_{2} \ddot{x}_{2}+k\left(x_{2}-x_{1}\right)+b_{0}\left(\ddot{x}_{2}-\ddot{x}_{3}\right)+b_{r}\left[\ddot{x}_{2}(t-\tau)-\ddot{x}_{3}(t-\tau)\right]=0 \\
& b_{0}\left(\ddot{x}_{2}-\ddot{x}_{3}\right)+b_{r}\left[\ddot{x}_{2}(t-\tau)-\ddot{x}_{3}(t-\tau)\right]=c\left(\dot{x}_{3}-\dot{x}_{1}\right)
\end{aligned}
$$

where $m_{1}$ is the unsprung mass, $m_{2}$ is the sprung mass, $x_{r}$ is the road random input, $x_{1}$ is the unsprung mass displacement, $x_{2}$ is the sprung mass displacement, $x_{3}$ is the two terminals displacement of the controllable inerter, $k_{t}$ is the stiffness of the tire, $k$ is the spring stiffness, $c$ is the coefficient of the damper, $t$ is time, $\tau$ is the time-delay. 


\subsection{Analyzing the stability theory of semi-active ISD suspension}

According to theory of linear differential equations with time-dependent constant coefficients (Babister, 1957; Qin et al., 1989), the general solution of homogeneous equations (3.3) and (3.4) is shown as

$$
x_{r}(t)=\left\{\begin{array}{lll}
X_{r} \mathrm{e}^{\lambda t} & r=1,2 & \text { for } \quad \mathrm{S} 1 \\
X_{r} \mathrm{e}^{\lambda t} & r=1,2,3 & \text { for } \quad \mathrm{S} 2
\end{array}\right.
$$

In which $X_{r}$ represents the solution of Laplace transform of the $x_{r}, \lambda$ is the eigenvalue, $t$ is time. Taking the general solution into Eq. (3.3), one deduces the characteristic equation corresponding to the homogeneous differential equations as shown in Eq. (3.6). Equation (3.6) is constantly equal to zero from differential Eq. (3.3)

$$
\left|\begin{array}{cc}
m_{1} \lambda^{2}+b_{0} \lambda^{2}+b_{r} \lambda^{2} \mathrm{e}^{-\lambda \tau}+c \lambda+k_{t}+k & -b_{0} \lambda^{2}-b_{r} \lambda^{2} \mathrm{e}^{-\lambda \tau}-c \lambda-k \\
-b_{0} \lambda^{2}-b_{r} \lambda^{2} \mathrm{e}^{-\lambda \tau}-c \lambda-k & m_{2} \lambda^{2}-b_{0} \lambda^{2}-b_{r} \lambda^{2} \mathrm{e}^{-\lambda \tau}-c \lambda+k
\end{array}\right|=0
$$

According to the Lyapunov stability criterion of invariant linear differential equations with constant coefficients (Wen et al., 2015; Grigoryan, 2015), the sufficient and necessary condition for the asymptotic stability of the system with time-delay is that all the eigenroots of characteristic Eq. (3.6) lie in the left portion of the complex plane. The marginally stable condition of the system is that the eigenroot of Eq. (3.6) has just imaginary roots $\lambda$. Suppose that $\lambda=j \omega$, the system will demonstrate self-excited vibration for the fundamental frequency of the natural frequency $\omega$. Taking the imaginary roots $\lambda$ into Eq. (3.6), and using Euler's formula, one separates the real and imaginary part of Eq. (3.6). The condition that only contains pure imaginary roots is illustrated as

$$
\begin{aligned}
& \Re=k k_{t}-b_{0} k_{t} w^{2}+b_{0} m_{1} w^{4}+b_{0} m_{2} w^{4}-k m_{1} w^{2}-k m_{2} w^{2}-k_{t} m_{2} w^{2}+m_{1} m_{2} w^{4} \\
& \quad-b_{r} k_{t} w^{2} \cos (\tau w)+b_{r} m_{1} w^{4} \cos (\tau w)+b_{r} m_{2} w^{4} \cos (\tau w)=0 \\
& \Im=c k_{t} w-c m_{2} w^{3}-c m_{1} w^{3}+b_{r} k_{t} w^{2} \sin (\tau w)-b_{r} m_{1} w^{4} \sin (\tau w)-b_{r} m_{2} w^{4} \sin (\tau w)=0
\end{aligned}
$$

with the identity $\sin ^{2}(\omega \tau)+\cos ^{2}(\omega \tau)=1$.

In order to obtain the value of the critical time-delay (Fu et al., 2007) $\tau$, Equation (3.5) should satisfy that all the roots are real. The simplest polynomial (3.8) with only self-excited vibration frequency $\omega$ is obtained as

$$
\frac{a_{4} \omega^{8}+a_{3} \omega^{6}+a_{2} \omega^{4}+a_{1} \omega^{2}+a_{0}}{a_{9} \omega^{8}+a_{8} \omega^{6}+a_{7} \omega^{4}+a_{6} \omega^{2}+a_{5}}=0
$$

In which

$$
\begin{aligned}
a_{0}= & k^{2} k_{t}^{2} \\
a_{1} & =c^{2} k_{t}^{2}-2 k^{2} k_{t} m_{1}-2 k k_{t}^{2} m_{2}-2 k^{2} k_{t} m_{2}-2 b_{0} k k_{t}^{2} \\
a_{2} & =b_{0}^{2} k_{t}^{2}+4 b_{0} k k_{t} m_{1}+4 b_{0} k k_{t} m_{2}+2 b_{0} k_{t}^{2} m_{2}-m_{2}^{2} k_{t}^{2}-2 c^{2} k_{t} m_{1}-2 c^{2} k_{t} m_{2}+k^{2} m_{1}^{2} \\
& +2 k^{2} m_{1} m_{2}+k^{2} m_{2}^{2}+4 k k_{t} m_{1} m_{2}+2 k k_{t} m_{2}^{2}+k_{t}^{2} m_{2}^{2} \\
a_{3}= & c^{2} m_{1}^{2}+c^{2} m_{2}^{2}-2 k m_{1} m_{2}^{2}-2 k m_{1}^{2} m_{2}-2 k_{t} m_{1} m_{2}^{2}-2 b_{0} k m_{1}^{2}-2 b_{0} k m_{2}^{2}-2 b_{0}^{2} k_{t} m_{1} \\
& -2 b_{0} k_{t} m_{2}^{2}-2 b_{0}^{2} k_{t} m_{2}+2 m_{2}^{2} k_{t} m_{1}+2 m_{2}^{2} k_{t} m_{2}+2 c^{2} m_{1} m_{2}-4 b_{0} k m_{1} m_{2}-4 b_{0} k_{t} m_{1} m_{2} \\
a_{4}= & b_{0}^{2} m_{1}^{2}+2 b_{0}^{2} m_{1} m_{2}+b_{0}^{2} m_{2}^{2}+2 b_{0} m_{1}^{2} m_{2}+2 b_{0} m_{1} m_{2}^{2}-m_{2}^{2} m_{1}^{2}-2 m_{2}^{2} m_{1} m_{2} \\
& -m_{2}^{2} m_{2}^{2}+m_{1}^{2} m_{2}^{2} \\
a_{5} & =0 \\
a_{8} & =-2 m_{2}^{2} k_{t} m_{1}-2 m_{2}^{2} k_{t} m_{2} \\
a_{9} & =m_{2}^{2} m_{1}^{2}+2 m_{2}^{2} m_{1} m_{2}+m_{2}^{2} m_{2}^{2}
\end{aligned}
$$


For a concrete kind of vehicle, $\omega$ and $\tau$ can be acquired through numerical calculation. What is more, the variation law of the critical instability time-delay of S1 structure with the base value of the inerter coefficient, the controllable inerter coefficient and the sprung mass were analyzed. The parameter values of the semi-active ISD suspension are shown in Table 1.

Table 1. The parameter values of semi-active ISD suspension

\begin{tabular}{|l|c|}
\hline \multicolumn{1}{|c|}{ Parameter } & Values \\
\hline \hline Spring stiffness $k[\mathrm{kN} / \mathrm{m}]$ & 22 \\
\hline Damping coefficient $c[\mathrm{kN} \mathrm{s} / \mathrm{m}]$ & 1.2 \\
\hline Tire stiffness $k_{t}[\mathrm{kN} / \mathrm{m}]$ & 190 \\
\hline Unsprung mass $m_{1}[\mathrm{~kg}]$ & 45 \\
\hline
\end{tabular}

To analyze the variation law of the critical instability time-delay $\tau$ of S1 structure with the base value of the inerter coefficient and the controllable inerter coefficient, the sprung mass $m_{2}$ was set as $320 \mathrm{~kg}$, the base value of the inerter coefficient $b_{0}$ and the controllable inerter coefficient $m_{2}$ were all set within 0 to $800 \mathrm{~kg}$. The root closest to the origin in the left part of the complex plane was used as the solution of the equation. The result is illustrated in Fig. 3.

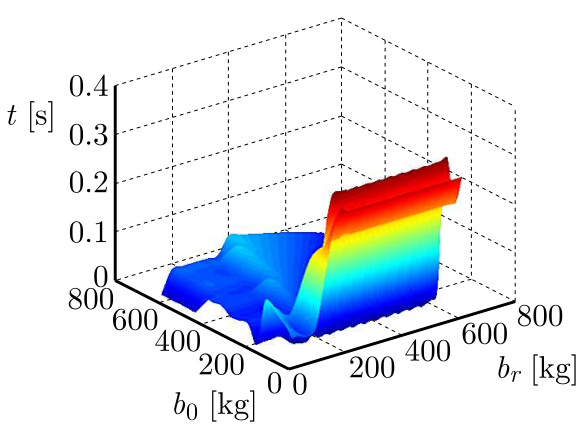

Fig. 3. The variation law of the critical instability time-delay of the semi-active ISD suspension with $b_{0}$ and $b_{r}$

This calculation result indicates that there is a large value of $\tau$ when the value of $b_{0}$ is small. The conclusions of other numerous researchers also show that $b_{0}$ should be smaller to get a better kinetic performance (Chen M.Z.Q. et al., 2016).

So, there are two methods to get a large critical time-delay:

Option 1: Select the greater value of the critical delay in the larger value of $b_{0}$ part.

When $m_{2}$ exceeded $250 \mathrm{~kg}$, the critical time-delay $\tau$ was stabilized at about $0.08 \mathrm{~s}$. When $m_{2}$ was about $350 \mathrm{~kg}$, the largest critical instability time-delay $\tau$ could be obtained.

Option 2: Select the small $b_{0}$. When $b_{0}$ was about $50 \mathrm{~kg}$, the critical instability time-delay $\tau$ reached about $0.27 \mathrm{~s}$.

In Option 2, the critical time-delay stability is better than in Option 1.

To further analyze the most suitable value of $b_{0}$ and search the variation law of the critical instability time-delay of the semi-active ISD suspension with the controllable inerter coefficient and the sprung mass, the base value of the inerter coefficient $b_{0}$ was set as $50 \mathrm{~kg}$; the sprung mass $m_{2}$ was set from $200 \mathrm{~kg}$ to $520 \mathrm{~kg}$; the controllable inerter coefficient $m_{2}$ was set from 0 to $800 \mathrm{~kg}$.

Figure 4 demonstrates that the small value of the sprung mass $m_{2}$ can enhance the performance. Luckily, the technology of lightweight automobile can help the light of the sprung mass $m_{2}$. So the parameter $b_{0}$ was about $50 \mathrm{~kg}, b_{r}$ was about $320 \mathrm{~kg}, m_{2}$ was as little as possible. 


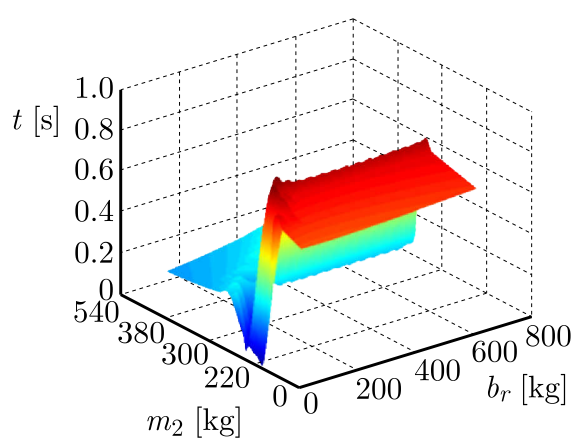

Fig. 4. The variation law of the critical instability time-delay of the semi-active ISD suspension with $m_{2}$ and $b_{r}\left(b_{0}=50 \mathrm{~kg}\right)$

Similarly, taking the general solution of Eq. $(3.5)_{2}$ into Eq. (3.4), one deduces the characteristic equation corresponding to the homogeneous differential equations as shown in Eq. (3.10). Equation (3.10) was constantly equal to zero from differential Eq. (3.4)

$$
\left|\begin{array}{ccc}
m_{1} \lambda^{2}+c \lambda+k_{t}+k & -k & -c \lambda \\
-k & m_{2} \lambda^{2}+b_{0} \lambda^{2}+b_{r} \lambda^{2} \mathrm{e}^{-\lambda \tau}+k & -b_{0} \lambda^{2}-b_{r} \lambda^{2} \mathrm{e}^{-\lambda \tau}-c \lambda \\
c \lambda & b_{0} \lambda^{2}+b_{r} \lambda^{2} \mathrm{e}^{-\lambda \tau} & -b_{0} \lambda^{2}-b_{r} \lambda^{2} \mathrm{e}^{-\lambda \tau}-c \lambda
\end{array}\right|=0
$$

Suppose that $\lambda=j \omega$, then the system demonstrates self-excited vibration for the fundamental natural frequency $\omega$. Imaginary roots $\lambda$ were taken into Eq. (3.10), and Euler formula was utilized to separate the real and imaginary part of Eq. (3.10). The condition of only imaginary root is illustrated as

$$
\begin{aligned}
\Re= & b_{0} c^{2} w^{4}-c^{2} k w^{2}+b_{0} k k_{t} w^{2}-b_{0} k m_{1} w^{4}-b_{0} k m_{2} w^{4}-b_{0} k_{t} m_{2} w^{4}+b_{0} m_{1} m_{2} w^{6} \\
& +b_{r} c^{2} w^{4} \cos (\tau w)+b_{r} k k_{t} w^{2} \cos (\tau w)-b_{r} k m_{1} w^{4} \cos (\tau w)-b_{r} k m_{2} w^{4} \cos (\tau w) \\
& -b_{r} k_{t} m_{2} w^{4} \cos (\tau w)+b_{r} m_{1} m_{2} w^{6} \cos (\tau w)-b_{r} c k w^{3} \sin (\tau w)-b_{r} c m_{2} w^{5} \sin (\tau w)=0 \\
\Im= & c k m_{1} w^{3}-c k k_{t} w-b_{0} c k w^{3}-b_{0} c m_{2} w^{5}-b_{r} c^{2} w^{4} \sin (\tau w)+c k m_{2} w^{3}+c k_{t} m_{2} w^{3} \\
& -c m_{1} m_{2} w^{5}-b_{r} c k w^{3} \cos (\tau w)-b_{r} c m_{2} w^{5} \cos (\tau w)-b_{r} k k_{t} w^{2} \sin (\tau w) \\
& +b_{r} k m_{1} w^{4} \sin (\tau w)+b_{r} k m_{2} w^{4} \sin (\tau w)+b_{r} k_{t} m_{2} w^{4} \sin (\tau w)-b_{r} m_{1} m_{2} w^{6} \sin (\tau w)=0
\end{aligned}
$$

Since the real and imaginary parts were all including $\sin (\tau w)$ and $\cos (\tau w)$ terms, the Taylor series expansion was used to solve Eqs. (3.11). To analyze the variation law of the critical instability time-delay $\tau$ of S2 structure with the base value of the inerter coefficient and the controllable inerter coefficient, the sprung mass $m_{2}$ and other parameters of the vehicle were the same as in $\mathrm{S} 1$ structure. The result is illustrated in Fig. 5.

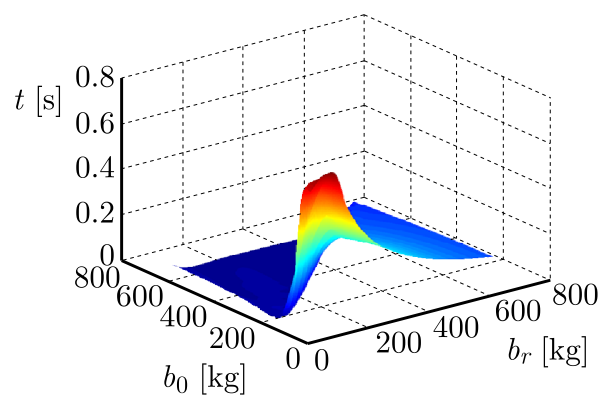

Fig. 5. The variation law of the critical instability time-delay of the semi-active ISD suspension with $b_{0}$ and $b_{r}$ 
The critical instability time-delay of S2 structure changes quickly with $b_{0}$ and $b_{r}$. When $b_{0}$ and $b_{r}$ were little, the critical instability time-delay $\tau$ was much larger than S1 structure. However, with addition to the value of $b_{0}$ and $b_{r}$, the critical instability time-delay $\tau$ was suddenly declined. And Table 2 shows significant differences of the critical time-delay with different parameters. Figure 6 is the variation law of the critical instability time-delay of S2 structure with $m_{2}$ and $b_{r}$ under the condition of $b_{0}=25 \mathrm{~kg}$. In this structure, it is guessed that the sprung mass has relatively little influence on the critical instability time-delay. And with a smaller controllable inerter coefficient $m_{2}$, the performance on time-delay stability of S2 structure is suitable. However, the time-delay of the control system is rarely over $0.5 \mathrm{~s}$. So, the system is not very demanding for the value of $m_{2}$.

Table 2. Some values of Fig. 5

\begin{tabular}{|c|c|c|c|c|}
\hline \multirow{2}{*}{$b_{0}[\mathrm{~kg}]$} & \multicolumn{4}{|c|}{$b_{r}[\mathrm{~kg}]$} \\
\cline { 2 - 5 } & 50 & 100 & 200 & 800 \\
\hline \hline 100 & 0.8216 & 0.7965 & 0.5155 & 0.2605 \\
\hline 200 & 0.6981 & 0.7505 & 0.4920 & 0.2578 \\
\hline 400 & 0.0403 & 0.0705 & 0.4024 & 0.2460 \\
\hline 800 & 0.0214 & 0.0072 & 0.0098 & 0.2012 \\
\hline
\end{tabular}

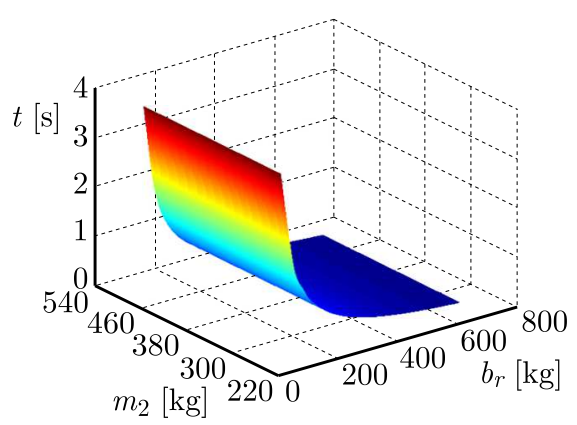

Fig. 6. The variation law of the critical instability time-delay of the semi-active ISD suspension with $b_{r}$ and $m_{2}$

Considering the time delay and comprehensive performance of the suspension, the suitable parameters of S1 structure were chosen as follows: $b_{0}$ was $50 \mathrm{~kg}, b_{r}$ was $200 \mathrm{~kg}$. The critical instability time-delay of S1 structure was $0.27 \mathrm{~s}$. And the suitable parameters of S2 structure were chosen as follows: $b_{0}$ was $25 \mathrm{~kg}, b_{r}$ was $120 \mathrm{~kg}$. The critical instability time-delay of $\mathrm{S} 2$ structure was $0.20 \mathrm{~s}$.

\section{Performance analysis of semi-active ISD suspension system}

To analyze the performance of the semi-active ISD suspension, random road information was taken as the input to the traditional suspension for comparison. The parameters of the traditional suspension were $k=22000 \mathrm{~N} / \mathrm{m}, c=1200 \mathrm{Ns} / \mathrm{m}$. The suspension parameters of S1 and S2 are exhibited in Table 3. The value of the inerter coefficient $b_{0}$ and $b_{r}$ were selected by the previous numerical analysis in Section 2. Other parameters are selected based on the experience and reference from some common passenger vehicles. To distinguish the inerter parameters between the two structures, the parameter of S1 structure was indicated by the index 01 and $r 1$. Structure 22 was indicated by the index 02 and $r 2$. 
Table 3. Suspension parameters of S1 and S2

\begin{tabular}{|l|c|}
\hline \multicolumn{1}{|c|}{ Parameter } & Values \\
\hline \hline Unsprung mass $m_{1}[\mathrm{~kg}]$ & 45 \\
\hline Sprung mass $m_{2}[\mathrm{~kg}]$ & 320 \\
\hline Spring stiffness $k[\mathrm{~N} / \mathrm{m}]$ & 22000 \\
\hline Tire stiffness $k_{t}[\mathrm{~N} / \mathrm{m}]$ & 190000 \\
\hline Damping coefficient $c[\mathrm{Ns} / \mathrm{m}]$ & 1200 \\
\hline Base value of inerter coefficient $b_{01}[\mathrm{~kg}]$ & 50 \\
\hline Base value of inerter coefficient $b_{02}[\mathrm{~kg}]$ & 25 \\
\hline Controllable inerter coefficient $b_{r 1}[\mathrm{~kg}]$ & 200 \\
\hline Controllable inerter coefficient $b_{r 2}[\mathrm{~kg}]$ & 120 \\
\hline
\end{tabular}

The system response was analyzed. Taking integral white noise of time-domain expression as the road input model ( $\mathrm{Yu}$ and Lin, 2005), the input equation was

$$
\dot{z}_{r}(t)=2 \pi \sqrt{G_{0} u} w(t)
$$

In which $w(t)$ is a mean of zero Gauss white noise, $u$ is the vehicle speed, $G_{0}$ is the road roughness coefficient. For the vehicle $u=20 \mathrm{~m} / \mathrm{s}$, the passing roughness factor was $G_{0}=5 \cdot 10^{-6} \mathrm{~m}^{3} /$ cycle. The output power spectral density with a random input for the traditional suspension, and structures S1, S2 are manifested in Table 4. Because the skyhook control strategy is comfort-oriented, the root mean square (RMS) value of body acceleration decreased under this control strategy. This means that the performance of riding comfort is improved by utilizing a controllable inerter. But the performance of suspension working space and dynamic tire load had a little deterioration or just little improved.

Table 4. RMS values of random response outputs

\begin{tabular}{|l|c|c|c|}
\hline \multicolumn{1}{|c|}{ Performance index } & Traditional suspension & S1 & S2 \\
\hline \hline Body acceleration RMS $\left[\mathrm{m} / \mathrm{s}^{2}\right]$ & 2.3464 & 1.5630 & 1.6886 \\
\hline Suspension working space RMS $[\mathrm{m}]$ & 0.0188 & 0.0232 & 0.0185 \\
\hline Dynamic tire load RMS $[\mathrm{kN}]$ & 1.5492 & 1.5716 & 1.6242 \\
\hline
\end{tabular}

Figures 7, 8 and 9 demonstrate a comparison of the suspension performance for structure S1 and S2 for a small time-delay $(0.01 \mathrm{~s})$ and the critical instability time-delay. The RMS values in random response outputs and their variation are demonstrated in Table 5.

Table 5. RMS values and their variation of S1 and S2 structures with different time-delays

\begin{tabular}{|l|c|c|c|c|c|}
\hline Time-delay $[\mathrm{s}]$ & RMS & $\mathrm{S} 1$ & Variation & $\mathrm{S} 2$ & Variation \\
\hline \hline \multirow{3}{*}{0 (No time-delay) } & Body acceleration & 1.5630 & - & 1.6886 & - \\
\cline { 2 - 6 } & Suspension working space & 0.0232 & - & 0.0185 & - \\
\cline { 2 - 6 } & Dynamic tire load & 1.5716 & - & 1.6242 & - \\
\hline \multirow{2}{*}{$\begin{array}{l}0.1 \text { (Little } \\
\text { time-delay) }\end{array}$} & Body acceleration & 1.5863 & $1.49 \%$ & 2.7601 & $63.45 \%$ \\
\cline { 2 - 6 } & Suspension working space & 0.0231 & $-0.43 \%$ & 0.0189 & $2.16 \%$ \\
\cline { 2 - 6 } $\begin{array}{l}\tau \text { (Critical } \\
\text { instability } \\
\text { time-delay) }\end{array}$ & Dynamic tire load & 1.5869 & $0.97 \%$ & 1.3587 & $-16.35 \%$ \\
\cline { 2 - 6 } & Buspension working space & 0.0227 & $-2.15 \%$ & 0.0202 & $9.19 \%$ \\
\cline { 2 - 6 } & Dynamic tire load & 1.6268 & $3.51 \%$ & 1.9762 & $21.67 \%$ \\
\hline
\end{tabular}

In Table 5, the positive variation number means that the value was increased and the performance was deteriorated. The negative variation number means that the value was decreased and 

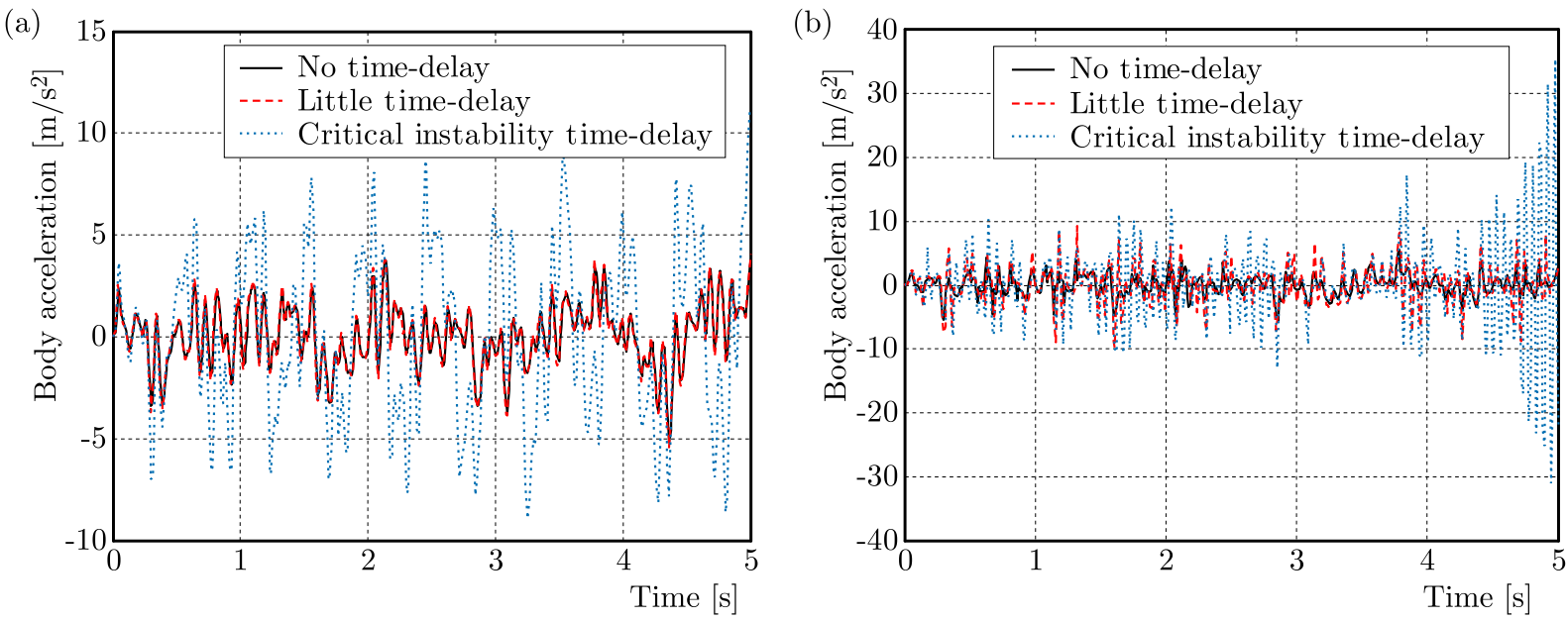

Fig. 7. Body acceleration with different time-delays; (a) S1 structure, (b) S2 structure
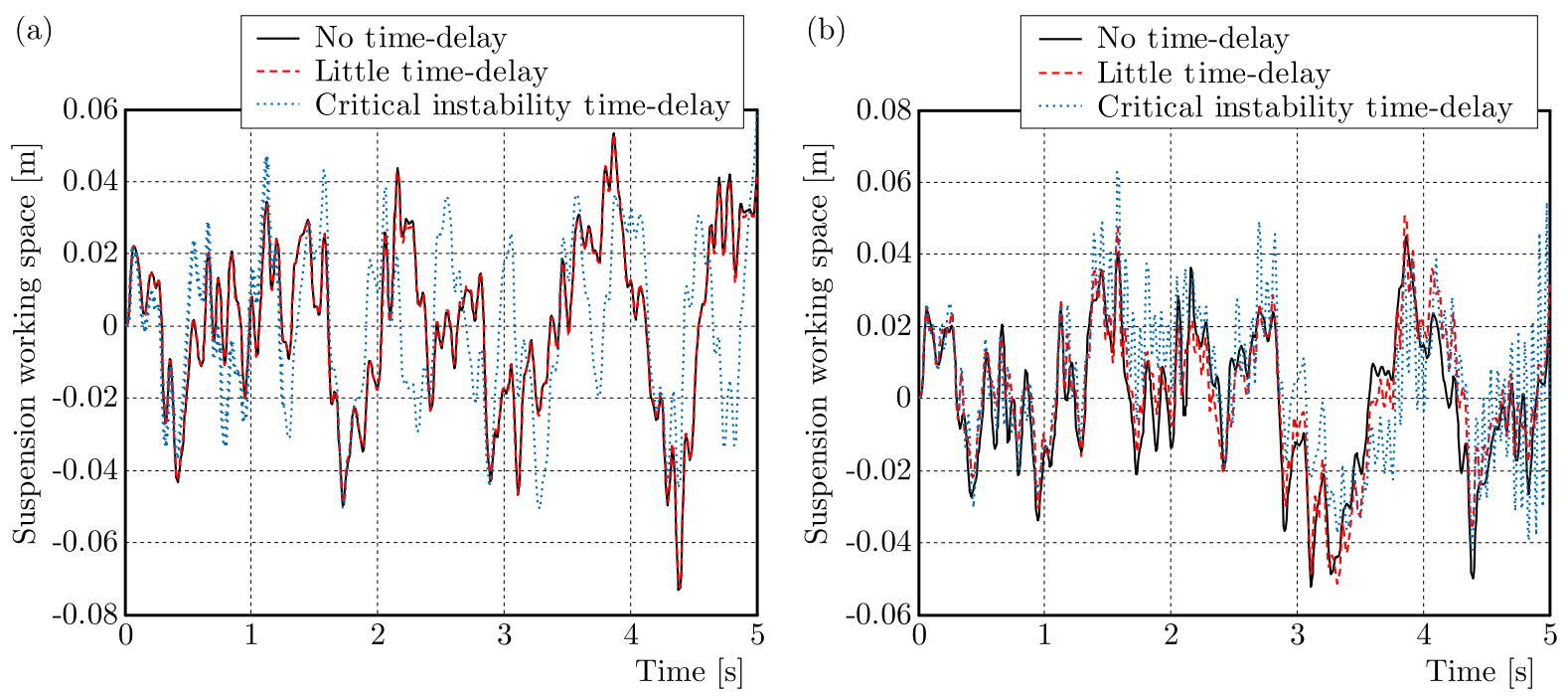

Fig. 8. Suspension working space with different time-delays; (a) S1 structure, (b) S2 structure
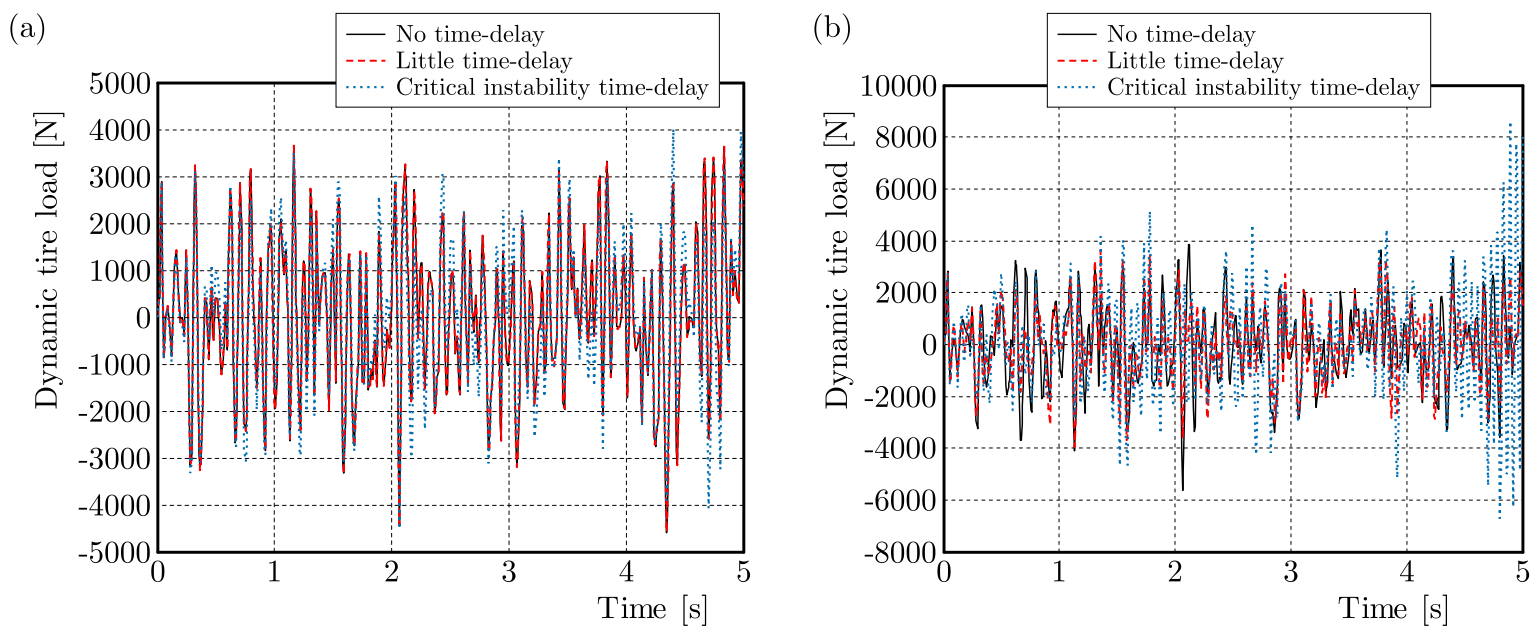

Fig. 9. Dynamic tire load with different time-delays; (a) S1 structure, (b) S2 structure 
the performance was enhanced. Figures 7-9 and Table 5 indicate that the little time-delay within the critical instability time-delay also deteriorates the suspension performance slightly. Furthermore, the performance of riding comfort deteriorates obviously with a surplus of the time-delay over the critical instability time-delay. This distinguishing exacerbation of the function leads to failure of the control system under the condition of the large time-delay.

\section{Conclusion}

- A general solution to stability analysis was proposed and utilized in analyzing the variation law of the critical instability time-delay in different semi-active ISD suspension structures. There is a significant impact upon the stability of the system with a time-delay. This paper provided a method to select suitable parameters in semi-active suspension design to avoid negative effects of the time-delay.

- The kinetic and stability of two three-element-structure ISD suspensions were analyzed from the perspective of the time-delay. Results suggest that different structures contain different kinetic features. The placing of the inerter greatly enriched the suspension characteristics.

- Two three-element-structure semi-active ISD suspensions effectively inhibit vertical vibration of the vehicle and improve the ride comfort. Although the time response of the inerter force control is shorter than the damping force control, a little time-delay will deteriorate the performance of the semi-active ISD suspension. And a large time-delay which exceeds the critical instability time-delay will obviously deteriorate the performance of the semiactive ISD suspension. So, how to avoid or reduce the time-delay is the significant problem in the suspension design.

\section{Acknowledgment}

This research has been supported by the National Natural Science Foundation of China (grant No. 51405202), Natural Science Foundation of Jiangsu Province (grant No. BK20160533), Scientific Research Innovation Projects of Jiangsu Province (grant No. KYLX15_1081), Postgraduate Education Reform Project of Jiangsu Province (KYCX18_2228).

\section{References}

1. BABISTER A.W., 1957, Stability and response of systems satisfying a second-order linear differential equation with time-dependent coefficients, Aeronautical Quarterly, 8, 1, 78-86

2. Chen L., Liu C., Liu W., Nie J., Shen Y., Chen G., 2017, Network synthesis and parameter optimization for vehicle suspension with inerter, Advances in Mechanical Engineering, 9, 1, 1-7

3. Chen L., Yang X., Wang R., Huang C., Shen Y., 2014, A study on the performances of vehicle passive suspension with modified inerter-spring-damper three-element structure, Automotive Engineering, 36, 3, 340-345

4. Chen M.Z.Q., Hu Y., Du B., 2012, Suspension performance with one damper and one inerter, Control and Decision Conference (CCDC), 2012 24th Chinese, IEEE, 3534-3539

5. Chen M.Z.Q., Hu Y., Li C., Chen G., 2016, Application of semi-active inerter in semi-active suspensions via force tracking, Journal of Vibration and Acoustics, 138, 4

6. Chen M.Z.Q., Smith M.C., 2015, Mechanical networks comprising one damper and one inerter, Control Conference, IEEE, 4917-4924

7. Eller D., Aggarwal J., Banks H., 1969, Optimal control of linear time-delay systems, IEEE Transactions on Automatic Control, 14, 6, 678-687

8. Estrada A., Efimov D., Perruquetti W., 2014, Position and velocity estimation through acceleration measurements, IFAC Proceedings Volumes, 47, 3, 6460-6465 
9. Fu P., Chen J., Niculescu S.I., 2007, High-order analysis of critical stability properties of linear time-delay systems, American Control Conference, IEEE, 4921-4926

10. Gonzalez-Buelga A., Clare L.R., Neild S.A., Jiang J.Z., Inman D.J., 2015, An electromagnetic inerter-based vibration suppression device, Smart Material Structures, 24, 5

11. Grigoryan G.A., 2015, On the stability of systems of two first-order linear ordinary differential equations, Differential Equations, 51, 3, 283-292

12. Han S.Y., Zhang C.H., TAng G.Y., 2017, Approximation optimal vibration for networked nonlinear vehicle active suspension with actuator time delay, Asian Journal of Control, 19, 3

13. Hu Y., Chen M.Z.Q., Xu S.. Liu Y., 2016, Semiactive inerter and its application in adaptive tuned vibration absorbers, IEEE Transactions on Control Systems Technology, 25, 1, 294-300

14. Hu Y., WAng K., Chen Y., Chen M.Z.Q., 2018, Inerter-based semi-active suspensions with low-order mechanical admittance via network synthesis, Transactions of the Institute of Measurement and Control, 40, 15, 4233-4245

15. Jiang J.Z., Smith M.C., 2015, Regular positive-real functions and passive networks comprising two reactive elements, Control Conference, IEEE, 2015, 219-224

16. KęCiK K, WARmiński J., 2011, Dynamics of an autoparametric pendulum-like system with a nonlinear semiactive suspension, Mathematical Problems in Engineering, 725, 34-35

17. Qin Y.-X., LiU Y.-Q., Wang L., 1989, Stable Dynamical Systems with Time Delays, 2nd ed., BeiJing, Science Press

18. SAPIŃski B., 2005, Fuzzy control for MR damper in a driver's seat suspension, Journal of Theoretical and Applied Mechanics, 43, 1, 179-201

19. Shen Y., Chen L., Liu Y., Zhang X., 2017, Analysis of vibration transfer characteristics of vehicle suspension system employing inerter, Journal of Theoretical and Applied Mechanics, 55, 4, $1245-1256$

20. Sipahi R., Olgac N., 2006a, Stability map of systems with three independent delays, 2006 American Control Conference, IEEE, DOI: 10.1109/ACC.2006.1656588

21. Sipahi R., Olgac N., 2006b, Stability robustness of retarded LTI systems with single delay and exhaustive determination of their imaginary spectra, SIAM Journal on Control and Optimization, 45, 5, 1680-1696

22. Sмiтh M.C., 2002, Synthesis of mechanical networks: the inerter, IEEE Transactions on Automatic Control, 47, 10, 1648-1662

23. Strecker Z., Mazưrek I., Roupec J., Klapka M., 2015, Influence of MR damper response time on semiactive suspension control efficiency, Meccanica, 50, 8, 1949-1959

24. Sun X., Chen L., Jiang H., Yang Z., Chen J., Zhang W., 2016, High-performance control for a bearingless permanent-magnet synchronous motor using neural network inverse scheme plus internal model controllers, IEEE Transactions on Industrial Electronics, 63, 6, 3479-3488

25. Vyhlidal T., Zitek P., 2009, Modification of Mikhaylov criterion for neutral time-delay systems, IEEE Transactions on Automatic Control, 54, 10, 2430-2435

26. Wen Y., Zhou X.F., Zhang Z., Liu S., 2015, Lyapunov method for nonlinear fractional differential systems with delay, Nonlinear Dynamics, 82, 1-2, 1015-1025

27. Yin X., Zhang L., Zhu Y., Wang C., Li Z., 2016, Robust control of networked systems with variable communication capabilities and application to a semi-active suspension system, IEEE/ASME Transactions on Mechatronics, 21, 4, 2097-2107

28. Yu F., Lin Y., 2005, Vehicle System Dynamics, Beijing, China Machine Press

29. Zuo L., Slotine J.J.E., 2005, Robust vibration isolation via frequency-shaped sliding control and modal decomposition, Journal of Sound and Vibration, 285, 4, 1123-1149 